\title{
Midline thalamic lesions enhance conditioned bradycardia and the cardiac orienting reflex in rabbits
}

\author{
SHIRLEY L. BUCHANAN and RICHARD H. THOMPSON \\ Wm. Jennings Bryan Dorn VA Medical Center, Columbia, South Carolina \\ and University of South Carolina, Columbia, South Carolina \\ and \\ D. A. POWELL \\ Wm. Jennings Bryan Dorn VA Medical Center, Columbia, South Carolina \\ and University of South Carolina School of Medicine, Columbia, South Carolina
}

\begin{abstract}
Learned heart rate changes were assessed during two sessions of differential Pavlovian (classical) conditioning in three groups of New Zealand albino rabbits. One group received ibotenic acid lesions of the midline nuclei of the thalamus, a second received vehicle injections in the midline nuclei, and a third group served as unoperated control animals. During an initial session, the cardiac component of the orienting reflex was assessed in all three groups. The rabbits with lesions of midline thalamic nuclei demonstrated enhanced bradycardiac conditioned responses, relative to either vehicle-control or unoperated animals. The heart rate component of the orienting response to nonreinforced tones, which also consisted of stimulus-evoked bradycardia, was likewise larger in lesioned animals. These results suggest that the midline thalamic nuclei, like the interconnected mediodorsal thalamic nucleus, may be involved in the regulation of sympatheticaliy mediated autonomic adjustments.
\end{abstract}

The midline thalamic nuclei in the rabbit include the paired paratenial (PT) and medioventral (MV) nuclei, and the unpaired paraventricular (PAV), rhomboid (RH), intermediodorsal (IMD), and reuniens (RE) nuclei. In addition, the centromedian nucleus, which is the most medial of the intralaminar nuclei, is often considered to be a part of the midline group because of its obvious midline position. This area of the thalamus, along with the more laterally situated mediodorsal (MD) nucleus, has been implicated by various investigators in certain aspects of learning (e.g., Aggleton \& Mishkin, 1983; Markowitsch, 1982; Squire, 1981). Likewise, several lines of evidence have suggested that the medial thalamus, including MD as well as the midline nuclei, may be involved in some aspect of autonomic function, specifically sympathetic function (Huang, Varner, Barman, \& Gebber, 1988; Varner, Barman, \& Gebber, 1988; West \& Benjamin, 1983).

Brutus et al. (1984) have suggested that MD may mediate autonomic adjustments through its connections with the midline thalamic nuclei, which in turn project to autonomic regulatory centers. These investigators specifi-

\footnotetext{
This research was supported by VA Institutional Research Funds awarded to the Wm. Jennings Bryan Dorn VA Medical Center. We thank the Medical Illustration Department of the University of South Carolina School of Medicine and the Medical Media Service of the VA Medical Center for assistance in preparation of the figures. We also thank Elizabeth Hamel for expert secretarial assistance. Correspondence may be addressed to Shirley L. Buchanan, Neuroscience Laboratory (151A), Wm. Jennings Bryan Dorn VA Medical Center, Columbia, SC 29201.
}

cally reported that, in the rat, nucleus reuniens showed increased $\left({ }^{14} \mathrm{C}\right) 2$-deoxyglucose (2DG) uptake following stimulation in MD. Nucleus reuniens stimulation, in turn, caused increased $\left({ }^{14} \mathrm{C}\right) 2 \mathrm{DG}$ activity in areas of the hypothalamus known to be involved in autonomic regulation. In the rabbit, stimulation of MD, which elicits primarily sympathetically mediated cardiovascular changes (Buchanan \& Powell, 1986), evokes increased $\left({ }^{3} \mathrm{H}\right) 2 \mathrm{DG}$ activity in PAV, PT, and IMD, but not in RE, suggesting possibly different connections in rabbits and rats (Buchanan \& Powell, 1987). Furthermore, parasagittal knife cuts medial to MD, which sever connections between this nucleus and the midline nuclei, attenuate parasympathetically mediated, classically conditioned bradycardia in the rabbit (Buchanan \& Powell, 1989). This contrasts with the effects of ibotenic acid (IA) lesions of $M D$, or of knife cuts lateral to MD, which sever MD-prefrontal connections. Both of these manipulations lead to an enhancement of parasympathetic responding (Buchanan, 1988; Buchanan \& Powell, 1989). These findings thus also implicate MD in sympathetic activation, since damage to MD or its efferent prefrontal connections result in relative parasympathetic dominance. As noted, stimulation studies (Buchanan \& Powell, 1986; Powell \& Buchanan, 1986), as well as electrophysiological evidence (Huang et al., 1988; Varner et al., 1988), support this conclusion.

If MD mediation of sympathetic activation is due to its connections with the midline nuclei, as previously sug- 
gested (e.g., see above), damage to these nuclei should also produce exaggerated bradycardia in response to classical conditioning contingencies. In the present study, we sought to test this hypothesis by examining differential Pavlovian heart rate (HR) conditioning in rabbits with IA lesions restricted to the midline nuclei. Animals with such lesions, or vehicle injections, were compared with a group of unoperated control animals. The HR response of the animals to novel tone stimuli (i.e., the cardiac component of the orienting reflex) was also assessed, since this response is also a parasympathetically mediated bradycardiac response, which is affected by IA lesions of MD (Buchanan, 1988).

\section{METHOD}

\section{Animals}

The animals were male and female New Zealand albino rabbits obtained from a local USDA-licensed supplier. The rabbits were maintained in an animal facility accredited by the American Association for the Accreditation of Laboratory Animal Care. A 12-h (7:00 a.m.-7:00 p.m.) light/dark cycle was employed; food and water were available ad lib. All behavioral testing was conducted during the daylight portion of the light/dark cycle.

\section{Apparatus}

Experimental contingencies were controlled by a PDP-11 microcomputer supplemented by solid-state TTL logic programming devices. Physiological responses were recorded on a Grass Model 7 polygraph equipped with appropriate preamplifiers. Electric shock was delivered from TTL constant-current shock generators through stainless steel wound clips attached to the infraorbital skin. During the experiment, the animals were restrained in Plexiglas rabbit restrainers in ventilated, sound- and light-attenuating, commercial animal enclosures (Industrial Acoustics Co., Model AC1). Auditory stimuli were produced by solid-state tone-oscillator circuits.

\section{Surgery}

The animals were anesthetized with ketamine hydrochloride $(55 \mathrm{mg} / \mathrm{kg}$ ) and acepromazine $(6 \mathrm{mg} / \mathrm{kg})$, i.m. In addition to the surgical procedure, chronic electrodes for presenting the unconditioned stimulus (US) and for measuring the HR conditioned response (CR) (see below) were also implanted while the animals were anesthetized. Nine animals received IA lesions of MD. IA (Sigma) was dissolved in phosphate buffer with a pH of $7.4(20 \mathrm{mg} / \mathrm{ml})$. Lesion injections were made through a Hamilton $1-\mu l$ syringe. Three injections were made on each side of the brain (P2.0, $\mathrm{L} \pm 0.5, \mathrm{~V} 9.5$; P3.5, $\mathrm{L} \pm 0.25, \mathrm{~V} 9.0$; and P5.0, $\mathrm{L} \pm 0.25$, V10.8, with reference to bregma, the midline suture, and dura, respectively). Each injection consisted of $0.25 \mu \mathrm{l}$ (i.e., $5 \mu \mathrm{g}$ ) injected over a 20 -min period. The syringe was left in place for $10 \mathrm{~min}$ after completion of the injection to ensure that no leakage occurred upon withdrawal from the brain. Following surgery, the animals were treated prophylactically with bicillin and were allowed a 2-week recovery period before behavioral testing began. Vehicle-control animals $(n=9)$ had similar injections of the phosphate buffer vehicle solution alone. An additional 9 animals served as unoperated control animals.

\section{Procedure}

Stainless steel safety pins inserted subcutaneously over the left hind flank and right foreleg served as ECG recording electrodes. Leads from these pins were connected to a Grass Model 7P4 EKG/tachograph preamplifier. Interbeat intervals (IBIs) were recorded, in milliseconds, by triggering a Schmidt trigger with the
R-wave. IBIs were recorded for 10 beats prior to tone onset and for the duration of the tone. The subjects received 2 days of differential Pavlovian conditioning, during which tones served as conditioned stimuli (CSs) and an AC paraorbital shock train served as the US. A $304-\mathrm{Hz}$ tone served as CS +, which was paired with the US, for half the animals, and a 1216- $\mathrm{Hz}$ tone served as $\mathrm{CS}+$ for the remaining animals. The other tone was the nonreinforced CS-, respectively, for each group. Each session consisted of $25 \mathrm{CS}+$ trials and $25 \mathrm{CS}$ - trials presented in a predetermined pseudorandom sequence. The intertrial interval was variable, ranging from 45 to $135 \mathrm{sec}$, with an average of $90 \mathrm{sec}$. Tone duration was $4 \mathrm{sec}$, and tone offset coincided with shock US onset. The US was a 0.25 $\mathrm{sec}, 2-$ to 3-mA, 60- $\mathrm{Hz}$ AC electric shock train delivered paraorbitally through the previously implanted stainless steel wound clips as noted above. On the day preceding the beginning of Pavlovian conditioning, the HR component of the orienting reflex (OR) in response to novel stimuli was assessed by presenting 10 tone-alone presentations. The tone used was one of the two tones to be used during conditioning, counterbalanced across subjects and to-be$\mathrm{CS}+$. HR in IBIs was recorded as discussed above. IBIs were recorded for 10 beats prior to tone onset and for the duration of the tone. For both the conditioning and the OR experiments, each IBI in milliseconds was converted to HR in beats per minute (bpm). Furthermore, the 10 pretone intervals on each trial were averaged to yield one baseline $H R$ value.

\section{Histology}

At the conclusion of testing, the animals were sacrificed with pentobarbital and perfused intracardially with physiological saline and $10 \%$ formalin. Forty-micrometer sections through the lesions (or the injection site for vehicle animals) were stained with thionin and projected onto plates from the atlas of Urban and Richard (1972). The extent of the lesion was determined by reference to a millimeter grid structure superimposed on these plates.

\section{RESULTS}

\section{Histology}

Histological analysis of the brain sections of the 9 animals with IA lesions revealed bilateral neuronal degeneration in midline nuclei in all animals. Figure 1 shows photomicrographs of representative sections through the thalamus in a lesioned animal. The top photograph is a low-magnification $(8 \times)$ photomicrograph of a section demonstrating the area damaged in the midline. The midline area, indicated by the closed arrow, can be seen to be cell-free, except for an area in dorsal PAV (open arrow). A higher power photomicrograph $(125 x)$ is shown in the bottom plate. This illustrates the extreme lateral edge of the lesion in the IMD nucleus. It can be seen that this area of IMD consists of a completely cell-free area in which considerable glial proliferation has occurred. Intact neurons can be seen on the right in MD. The neuronal degeneration seen in Figure 1 was typical of the damage seen in all the midline nuclei. In 3 animals, the damage extended into MD unilaterally, and 1 animal had bilateral damage to the extreme medial edge of MD. In 2 animals, unilateral anteromedial (AM) damage was observed, and in 2 animals the extreme medial border of AM was damaged bilaterally. One animal with bilateral AM damage also revealed unilateral damage to the anteroventral (AV) nucleus. In all cases, however, damage 

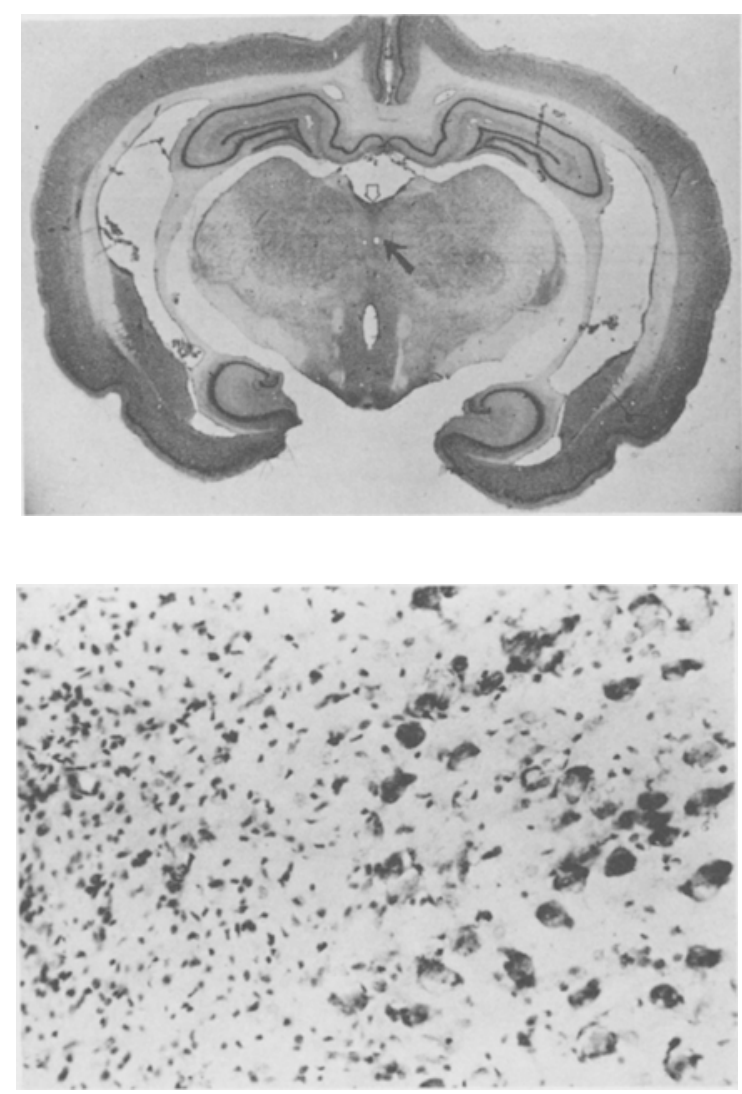

Figure 1. (Top) Photomicrograph of a brain section through midline thalamic lesion produced by ibotenic acid (thionin stain, $8 \times$ ). The lesion includes intermediodorsal and ventral paraventricular nuclei. (Bottom) Photomicrograph of the extreme lateral edge of the lesion (thionin stain, 125x). Lesions were characterized by an absence of cell bodies and increased glial formation (left). Intact neurons in the mediodorsal nucleus can be seen on the right.

to the nonmidline nuclei was minimal, and in the remaining animals, the damage was restricted to the midline nuclei. Figure 2 represents the maximal and minimal extent of the lesions, in the IA group, at 4 different anteriorposterior levels. Except for the extreme dorsal portions of PAV and IMD at anterior levels, the stippled area indicates the intersection of the damage that occurred in all 9 animals with IA lesions and the hatched area indicates the damage that occurred in at least 1 , but not all, animals. In 3 animals with lesions, the most extreme dorsal border of PAV and anterior IMD were not damaged (e.g., see Figure 1, top plate). However, to prevent unduly complicating the top portions of Figure 2, this minimal intact area in these 3 animals is not indicated. The brains of vehicle animals were also examined histologically but showed no neuronal degeneration, although the cannula tracks were localized to the midline region in all animals. Damage along the cannula track was minimal in all animals, and was comparable in lesioned and vehicle animals. No neuronal degeneration was seen along the track, in either the hippocampus or the overlying neocor- tex, indicating that no leakage occurred along the barrel of the cannula.

\section{Orienting}

The HR component of the OR was a cardiodeceleration in all animals, which declined in magnitude across the 10 trials. Figure 3 shows the mean HR change from pretone baseline for each of the 12 posttone intervals for the first, fifth, and last trials of the session for IA-lesioned, vehicle, and unoperated animals. Only the first 12 intervals are depicted, since for several animals (i.e., those with slower HRs) no further data were recorded; thus, the 12th interval was the last interval for which all animals had data. Figure 3 suggests that the HR OR was larger in the animals with IA lesions, particularly on the early trials. Data from this portion of the experiment were analyzed using analysis of variance (ANOVA). Difference scores (i.e., HR change scores) were generated by subtracting the HR associated with each posttone interval
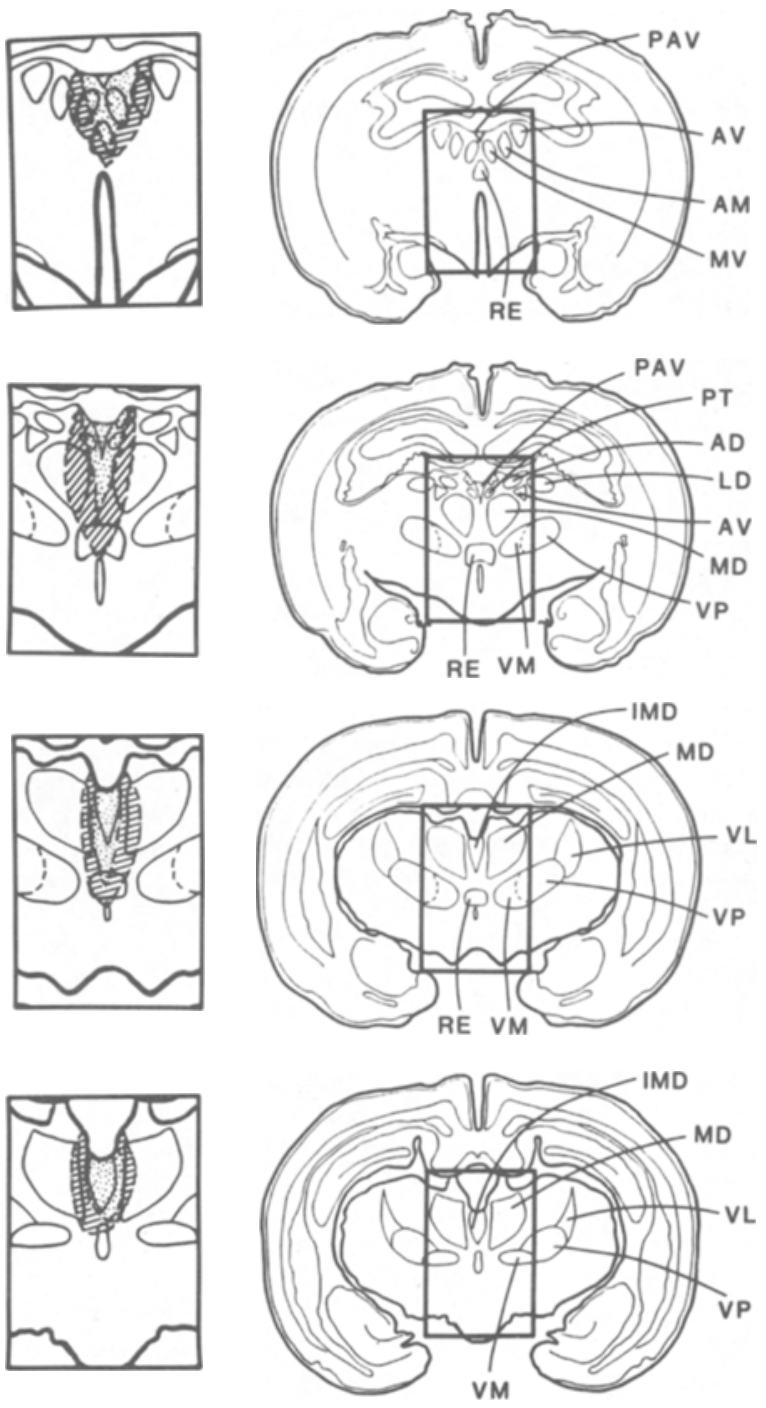

Figure 2. Maximal (hatched) and minimal (stippled) extent of damage in ibotenic acid-lesioned animals. Abbreviations as in text. 

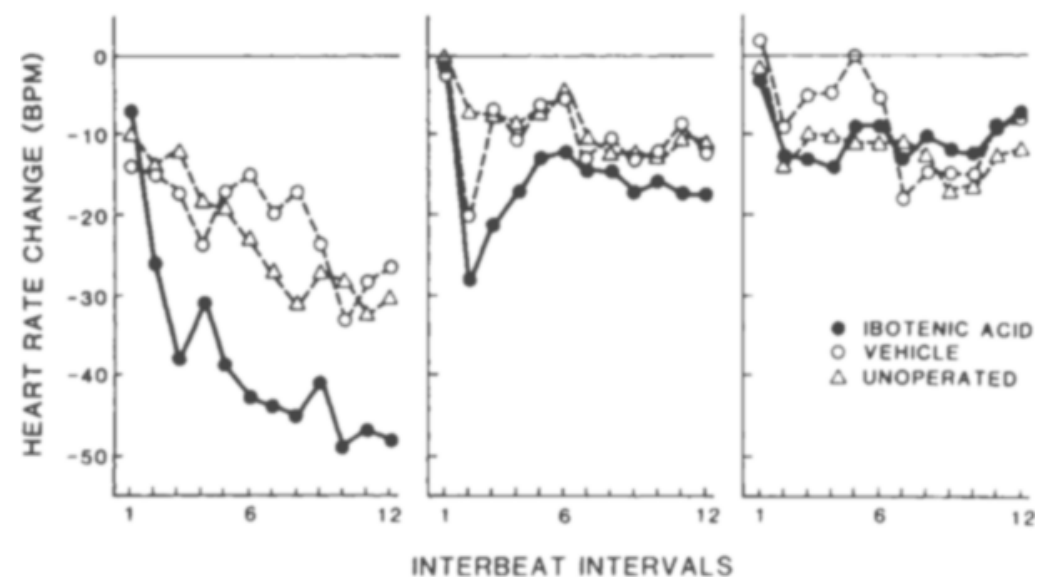

Figure 3. Mean heart rate change from baseline in response to nonreinforced tone presentations. Data are shown for each of 12 posttone interbeat intervals, for the 1st, 5th, and 10th tone presentations, for ibotenic acid-lesioned, vehicle, and unoperated animals.

from the pretone measure. Separate analyses were conducted on the baseline HR and on HR change, utilizing as dimensions group (3 levels), trial (10 levels), and, for the difference-score analysis, interval (12 levels). For the baseline measure, only the trial effect was significant $[F(9,172)=8.41, p<.0001]$, reflecting a decline in baseline HR across trials in all animals. Mean baseline HR $( \pm S E M)$ was $212.9 \pm 19.9,226.9 \pm 21.3$, and 241.0 \pm 33.4 for the lesion, vehicle, and unoperated animals, respectively, at the beginning of the session, but was 204.8 $\pm 25.3,220.1 \pm 35.4$, and $229.2 \pm 31.9$ by the last trial. This is a common finding and has been previously reported (e.g., Albiniak \& Powell, 1980).

The analysis of the difference scores yielded significant effects for trial, interval, and trial $\times$ interval, reflecting the reliability of the change from baseline across intervals and the habituation of the response across trials [smallest $F(126,2244)=2.32, p<.0001$, for trial $\times$ interval]. In addition, this analysis showed that there was a significant group $\times$ trial $\times$ interval interaction $[F(252,2244)=1.2, p<.02]$, suggesting that the differential pattern of responding seen in Figure 3 was reliable. Duncan posttests conducted on these data $(\alpha=$ $.05)$ showed that, on the early trials (i.e., Trials $1-3$ ), the IA animals showed larger decelerations than did either the vehicle or the unoperated control animals. This difference continued on certain intervals through Trial 7 (i.e., Trials 5,6, and 7), as can be seen in the middle panel of Figure 3. By the last trials in the session (i.e., Trials 8-10), there were no significant differences among the three groups. Thus, the animals with IA lesions showed an enhanced bradycardiac response to novel tone stimuli, which nonetheless habituated relatively normally.

\section{Conditioning}

As with the orienting data, the conditioning data were transformed from IBI to HR in beats per minute prior to data analysis. Further data reduction was carried out by averaging each interval, across five-trial blocks, for each session, subject, and CS. This yielded five trial blocks each for CS + and CS - . The HR CR was a deceleration from baseline, which reached a maximum of 20-30 beats early during Session 1 . The HR change over post-CS intervals is illustrated in Figure 4. This figure shows differ-

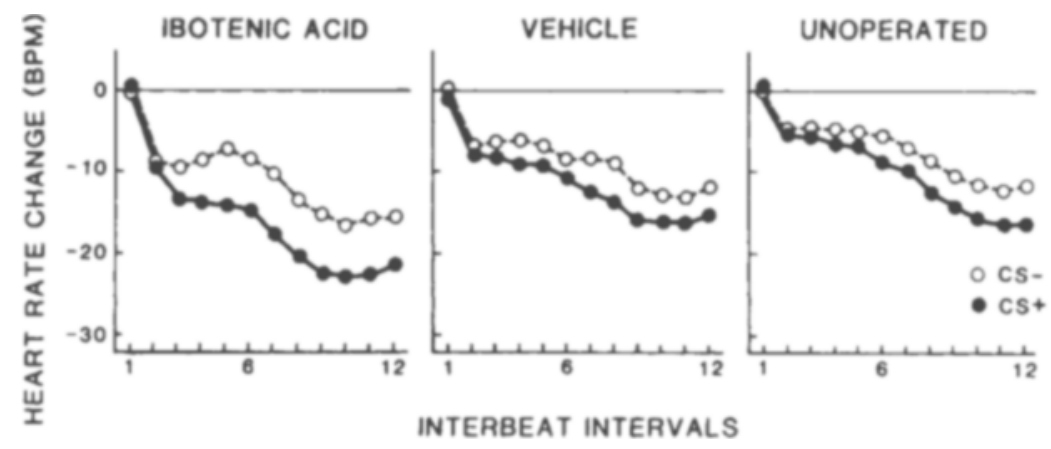

Figure 4. Mean heart rate change from pre-CS baseline during each of 12 post-CS interbeat intervals. Data shown represent the mean of the two sessions, for CS + and CS-, in ibotenic acid-lesioned, vehicle, and unoperated animals. 
ences in HR topography after tone onset pooled across the trials and session dimensions, since significant group interactions did not occur for these two variables (see below). Examination of this figure reveals that, as with orienting, the response of the IA animals was enhanced relative to the other two groups. Analysis was carried out by ANOVA utilizing as dimensions group ( 3 levels), session (2 levels), trial block (5 levels), CS (2 levels), and interval (12 levels). ANOVA revealed a significant interval effect $[F(11,264)=29.71, p<.0001]$, indicating that the HR change from baseline was reliable, as well as significant trial block $[F(4,96)=2.81, p<.03]$ and CS $[F(1,24)=32.76, p<.0001]$ effects, reflecting the development of the $C R$ across trials and the reliability of the HR discrimination. These effects were also reflected in significant interactions among trial block, interval, session, and CS [smallest $F(44,1045)=1.78, p<.001$, for trial $\times$ CS $\times$ interval]. In addition, there was a significant group $\times$ CS $\times$ interval effect $[F(22,264)=1.84$, $p<.01]$. Duncan posttests conducted on the data support the conclusion that the animals with IA lesions showed larger magnitude responses than did the vehicle or unoperated control groups, particularly to the CS+.
On Intervals 4-12, the animals with IA lesions showed significantly larger CS + responses than did the two control groups. In addition, on some intervals, these animals showed larger CS - responding than did the vehicle or the unoperated animals.

A separate analysis conducted on the baseline HR showed that the only significant group or group interaction effect involved the three-way interaction between group, session, and trial $[F(8,92)=2.79, p<.008]$. This was due to a significantly greater decline in baseline HR from Session 1 to Session 2 in the vehicle-control group relative to the other two groups. Mean baseline HR was $209.0 \pm 17.8,224.8 \pm 30.4$, and $225.5 \pm 25.3$ for the lesion, vehicle, and unoperated groups, respectively.

Although not apparent in Figure 4, it should be noted that the pattern of responding in many of the IA animals in the present experiment was different from that of both of the other groups, as well as from animals with MD damage as reported in a previous paper (Buchanan, 1988). In general, an individual rabbit's response on any given trial usually approximated the form depicted in Figure 4, that is, a fairly rapid deceleration beginning during the first two to three posttone intervals, which reached its
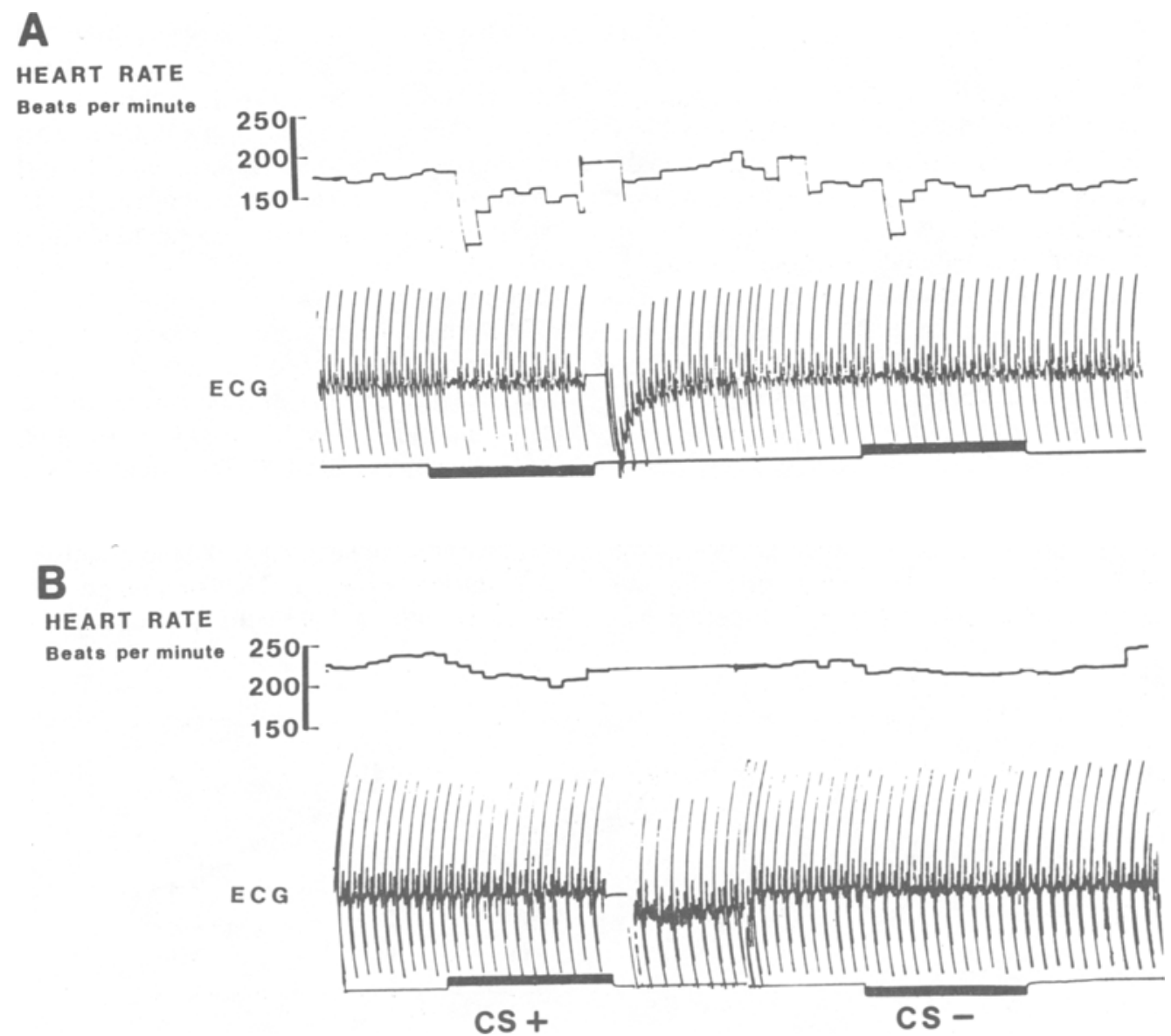

Figure 5. Tachograph recordings of heart rate change during the conditioning stimulus in (A) a representative animal with ibotenic acid lesions of the midline thalamic nuclei and (B) an animal with vehicle injections in the midline thalamic nuclei. 
maximum by Intervals $9-10$, followed by a gradual return toward baseline (seen in Intervals 11-12 in Figure 4). In animals with midline damage, however, the change from baseline was often much less gradual, and typically involved a rapid and dramatic drop in HR immediately after tone onset. HR changes of up to $100 \mathrm{bpm}$ occurred within 1-2 post-CS IBIs in many of these animals, as illustrated in Figure 5.

\section{DISCUSSION}

In the present experiment, lesions restricted to the midline thalamic nuclei mimicked the effects of IA lesions to MD in that there was an overall increase in stimulusevoked parasympathetic responding. The pattern of responding in this experiment was, however, not identical to that obtained with IA lesions of MD. MD damage led to impaired discrimination due to overresponding to the nonreinforced $\mathrm{CS}$-. In the present experiment, however, discrimination was actually somewhat superior following IA lesions of the midline nuclei, due to larger than normal CS + responding. The HR component of the OR likewise was enhanced in IA-lesioned animals. Again, in contrast to animals with MD damage, midline-lesioned animals habituated normally; MD damage led to enhanced responding accompanied by slower habituation of the response.

As discussed above, previous investigations have examined the role of the medial thalamus in various behavioral functions; few, if any, of these studies, however, have concerned the midline nuclei alone. Most lesion studies of this area have included damage to MD, and most anatomical studies have focused on MD as the primary area of interest. Indeed, West, Jackson, and Benjamin (1979) and Brutus et al. (1984) have suggested that it is the MDto-midline connections that are critical for autonomic regulation, since the midline nuclei, unlike MD, have been demonstrated to send projections to subcortical structures known to be involved in such regulation. At first glance, the present results appear to support such an interpretation of midline thalamic function, that is, as serving to route information from MD downward. However, the results mentioned above involving knife cuts, which severed connections between MD and the midline nuclei, suggest that this picture may be somewhat more complicated. If damage restricted to either MD or the midline nuclei leads to an enhancement of parasympathetic responding, then disruption of connections between these two areas would be expected to have similar effects, if the midline nuclei serve only to route MD information to autonomic regulatory structures. In fact, severing such connections had the opposite effect, that is, an attenuation of the bradycardiac response. These findings suggest a more complex interaction between MD and midline nuclei function.

A further point regards the form of the response in animals with midline lesions. As noted above, these animals showed a more abrupt change in HR than did control animals, in addition to manifesting overall larger changes. This pattern of responding shares some characteristics with that seen following knife cuts medial to MD (Buchanan \& Powell, 1989). In those animals, a similar large, abrupt (during the first 1-2 intervals) deceleration was seen, but it was followed by an equally rapid return toward baseline, probably reflecting compensatory mechanisms. The latter response was, however, not observed in animals with midline lesions in the present study. Thus, the pattern of responding following midline lesions is similar in some respects to that seen after MD damage (i.e., larger magnitude) and in some respects to that seen after medial knife cuts (i.e., more abrupt changes). As noted above, on the basis of several lines of evidence (e.g., Buchanan \& Powell, 1986; Huang et al., 1988), these enhanced HR decelerations are presumed to be due to an interruption of sympathetic tone by midline lesions.

As discussed above, the medial thalamus, and specifically MD, has been implicated in learning and memory, as well as in autonomic function. The present findings of enhanced CR magnitude appear to represent a parasympathetic bias resulting from damage to central nervous system structures normally involved in producing sympathetic changes. Particularly since such enhanced responding occurred during development of the $O R$ as well as the CR, these effects may be considered performance factors, rather than effects on learning per se. This conclusion is supported by the finding that the discrimination of the animals with IA lesions was unimpaired. This finding contrasts with the results following MD damage, in which the discrimination between $\mathrm{CS}+$ and $\mathrm{CS}-$ was impaired, suggesting an overall increase in responsivity, which had an indirect impact on learning (Buchanan, 1988). In the present experiment, discrimination was unimpaired, a finding that suggests that learning was intact.

These considerations are, however, complicated by the fact that, in the rabbit, the demarcation between the most medial aspect of MD and the midline nuclei is much less clearly defined than in species such as the cat and the monkey. West et al. (1979) have suggested that, in the rabbit, the midline nuclei are well defined and are most homologous with medial MD in primates and cats, which show poor midline nuclei differentiation but much better MD development than do rabbits. It may therefore be somewhat more difficult to distinguish functional differences between the midline nuclei and MD in rabbits than in other species. Nevertheless, the present results suggest that the medial thalamus is indeed important for the regulation of certain aspects of cardiovascular responding that are elicited during learning tasks and, furthermore, that the midline nuclei may play a more direct and important role in such functions than was previously thought.

\section{REFERENCES}

Aggleton, J. P., \& Mishin, M. (1983). Memory impairments following restricted medial thalamic lesions in monkeys. Experimental Brain Research, 52, 199-209.

Albiniak, A. B., \& Powell, D. A. (1980). Peripheral autonomic mechanisms and Pavlovian conditioning in the rabbit (Oryctolagus 
cuniculus). Journal of Comparative \& Physiological Psychology, 94, 1101-1113.

Brutus, M., Watson, R. E., Shaikh, M. B., Siegel, H. E., WeIner, S., \& SiEgel, A. (1984). A $\left({ }^{14} \mathrm{C}\right) 2$-deoxyglucose analysis of the functional neural pathways of the limbic forebrain in the rat: 4. A pathway from the prefrontal cortical-medial thalamic system to the hypothalamus. Brain Research, 310, 279-293.

Buchanan, S. L. (1988). Mediodorsal thalamic lesions impair differential Pavlovian heart rate conditioning. Experimental Brain Research, 73, 320-328.

Buchanan, S., \& Powell, D. A. (1986). Electrical stimulation of anteromedial and mediodorsal thalamus elicits differential cardiovascular response patterns from conscious rabbits. Physiological Psychology, 14, 115-123.

Buchanan, S. L., Powell, D. A. (1987). ${ }^{3}$ H-2-deoxyglucose uptake following stimulation of cardioactive sites in insular cortex and mediodorsal nucleus of the thalamus in rabbits. Brain Research Bulletin, 19, 439-452.

Buchanan, S. L., Powell, D. A. (1989). Parasagittal thalamic knife cuts and cardiac changes. Behavioral Brain Research, 32, 241-253.

Huang, Z.-S., Varner, K. J., Barman, S. M., Gebber, G. L. (1988). Diencephalic regions contributing to sympathetic nerve discharge in anesthetized cats. American Journal of Physiology, 254, R249-R256.
Markowitsch, H. J. (1982). Thalamic mediodorsal nucleus and memory: A critical evaluation of studies in animals and man. Neuroscience \& Biobehavioral Reviews, 6, 351-380.

Powell, D. A., \& Buchanan, S. L. (1986). Autonomic changes elicited by chemical stimulation of the mediodorsal nucleus of the thalamus. Pharmacology, Biochemistry \& Behavior, 25, 423-430.

SQuiRE, L. R. (1981). Two forms of human amnesia: An analysis of forgetting. Journal of Neuroscience, 1, 635-640.

URBAN, U., RICHARD, P. A. (1972). A stereotaxic atlas of the New Zealand rabbit. Springfield, IL: Thomas.

Varner, K. J., Barman, S. M., \& Gebber, G. L. (1988). Cat diencephalic neurons with sympathetic nerve-related activity. American Journal of Physiology, 254, R257-R267.

WEST, C. H. K., \& BENJAMIN, R. M. (1983). Effects of stimulation of the mediodorsal nucleus and its projection cortex on heart rate in the rabbit. Journal of the Autonomic Nervous System, 9, 547-557.

West, C. H. K., Jackson, J. C., Benjamin, R. M. (1979). An autoradiographic study of subcortical forebrain projections from mediodorsal and adjacent midline thalamic nuclei in the rabbit. Neuroscience, 4, 1977-1988.

(Manuscript received December 28, 1988; revision accepted for publication May 5, 1989.)

\title{
Announcement
}

\author{
30th Annual Meeting of the Psychonomic Society \\ Atlanta, Georgia \\ November 17-19, 1989
}

The 30th Annual Meeting of the Psychonomic Society will be held in Atlanta, Georgia, November 1719, 1989. The meetings will begin Friday morning and continue until Sunday at noon. The headquarters hotel will be the Hyatt Regency.

The program and hotel reservation cards will be mailed to members and associates in September. A copy of the program will be published in the November issue of the Bulletin of the Psychonomic Society.

For further information, please contact the secretary-treasurer of the Society: Michael E. Rashotte, Department of Psychology, Florida State University, Tallahassee, FL 32306-1051 (Telephone: 904-644-2040; BITNET: Rashot@FSU). 